\title{
A HIERARCHICAL METHOD FOR POLYLINE APPROXIMATION DIGITAL SINGLE-VALUED CURVES USING MOVING-POINT UN STRUCTURE \\ mohamed alsaid
}

Abstract

The approximation method suggested compromises between two conflicting requirements the first is to smooth out small fluctuations of a curve while the second is to preserve sharp changes in that curve. The suggested method is applied on singlevalued curves resulting in polyline approximations of that curves.

The polyline approximation of a digital curve aims to reduce the data required to represent that curve as much as possible while keeping the shape of the original curve as clear as possible at a specified level of accuracy. This reduction in data is a prerequisite for higher level processing for curves in application fields.

The suggested method is experimented on three curves with different local and global features. The results are compared with that of the arc tree method. The comparison shows that the suggested method is more efficient in data reduction 